\title{
Pyridine-promoted Cyclization of Functionalized N-Silylated Boron-Nitrogen Compounds
}

\author{
Fanny Schurz and Martin Jansen \\ Max Planck Institute for Solid State Research, Heisenbergstraße 1, 70569 Stuttgart, Germany \\ Reprint requests to Prof. Dr. Dr. h. c. M. Jansen. Fax: +49(0)711689-1502. \\ E-mail: m.jansen@fkf.mpg.de
}

Z. Naturforsch. 2011, 66b, 1225 - 1230; received October 7, 2011

\begin{abstract}
Dichloroboryldisilylamines $\left[\mathrm{SiCl}_{m}\left(\mathrm{CH}_{3}\right)_{3-m}\right] \mathrm{N}\left[\mathrm{Si}\left(\mathrm{CH}_{3}\right)_{3}\right]\left(\mathrm{BCl}_{2}\right)(m=1-3)$ as well as chloromethylboryl-bis-(chlorodimethylsilyl)amine $\left[\mathrm{SiCl}\left(\mathrm{CH}_{3}\right)_{2}\right]_{2} \mathrm{~N}\left[\mathrm{BCl}\left(\mathrm{CH}_{3}\right)\right]$ form $1: 1$ adducts with pyridine (1-4). Those with $m=2$ and 3 have been converted into functionalized diazadiboretidine derivatives which are still coordinated by pyridine: $\left[\left(\mathrm{SiCl}_{m}\left(\mathrm{CH}_{3}\right)_{3-m}\right) \mathrm{NBCl} \cdot \mathrm{Py}\right]_{2}(\mathbf{5}: m=2, \mathbf{6}$ : $m=3$ ). Single-crystal X-ray diffraction structure analyses confirm the presence of planar, rhombusshaped, four-membered boron-nitrogen rings with tetra-coordinated boron atoms and nitrogenbonded, chlorine-functionalized silyl groups, for both compounds.
\end{abstract}

Key words: Silazane Cleavage, Decomposition, Cyclization, Diazadiboretidine Derivatives, Crystal Structures

\section{Introduction}

For accessing the $\mathrm{Si} / \mathrm{B} / \mathrm{N} / \mathrm{C}$ ceramic system many different molecular single-source precursors have been considered and investigated regarding the influence of their constitutions on the properties of obtained polymers and ceramics $[1,2]$. In this context, cyclic boron-nitrogen compounds including borazine derivatives are of great interest because they offer the opportunity to introduce rigid structure fragments into the ceramic network [3-9]. Beside the common six-membered cyclic systems, also boronnitrogen compounds with four- and eight-membered rings are known [10 - 19]. Typically, cyclic representatives form during cyclization of aminoboranes or iminoboranes $[10,11,20-22]$, whereas the ring size depends mainly on the space requirement of the substituents [23,24]. Molecules like dichloroboryldisilylamines $\left[\mathrm{SiCl}_{m}\left(\mathrm{CH}_{3}\right)_{3-m}\right] \mathrm{N}\left[\mathrm{Si}\left(\mathrm{CH}_{3}\right)_{3}\right]\left(\mathrm{BCl}_{2}\right)(m=$ 0 [21, 25, 26], 1, 2 [9] and 3 [8]) are converted at elevated temperatures by silazane cleavage into six-membered borazine derivatives $\left[\left(\mathrm{SiCl}_{m}\left(\mathrm{CH}_{3}\right)_{3-m}\right)\right.$ $\left.\mathrm{NB}\left(\mathrm{Cl}_{n}\left(\mathrm{CH}_{3}\right)_{1-n}\right)\right]_{3}(m=0, n=1[21,25,26] ; m=$ $1, n=0.185$ [9]; $m=2, n=0.111$ [9]; $m=3$, $n=0.366$ [8]). In cases of $m=1-3$ an undesired substituent exchange between boron-bonded chlorine atoms and methyl entities of silyl groups occurs [8,9], probably using the free coordination sites of the boron atoms. In order to suppress such undesired rearrange- ments, we have studied the thermally induced conversion of dichloroboryldisilylamines $\left[\mathrm{SiCl}_{m}\left(\mathrm{CH}_{3}\right)_{3-m}\right]$ $\mathrm{N}\left[\mathrm{Si}\left(\mathrm{CH}_{3}\right)_{3}\right]\left(\mathrm{BCl}_{2}\right)(m=1-3)$ and of $\left[\mathrm{SiCl}\left(\mathrm{CH}_{3}\right)_{2}\right]_{2}-$ $\mathrm{N}\left[\mathrm{BCl}\left(\mathrm{CH}_{3}\right)\right]$ in the presence of pyridine (Scheme 1).

\section{Results and Discussion}

The pyridine adducts $\left[\mathrm{SiCl}_{m}\left(\mathrm{CH}_{3}\right)_{3-m}\right] \mathrm{N}\left[\mathrm{Si}\left(\mathrm{CH}_{3}\right)_{3}\right]-$ $\left(\mathrm{BCl}_{2} \cdot \mathrm{Py}\right)(\mathbf{1}: m=1, \mathbf{2}: m=2, \mathbf{3}: m=3)$, and [SiCl$\left.\left(\mathrm{CH}_{3}\right)_{2}\right]_{2} \mathrm{~N}\left[\mathrm{BCl}\left(\mathrm{CH}_{3}\right) \cdot \mathrm{Py}\right]$ (4) are formed at room temperature and are stable in boiling toluene. Mass spectra have confirmed the presence of pyridine displaying its specific fragments next to those characteristic for the base-free educt molecules [8,9]. However, the IR spectra are more complex due to additional vibrations of the pyridine ring in combination with distinct shifts of typical band positions. For example, the B-N stretching modes move from about $1230-$ $1300 \mathrm{~cm}^{-1}$ in the base-free compounds to lower wave numbers at $1079-1091 \mathrm{~cm}^{-1}$ in the corresponding adducts. The coordination of boron by pyridine results in a high-field shift of the respective NMR signals, especially ${ }^{11} \mathrm{~B}(\sim 40 \mathrm{ppm})$ and ${ }^{29} \mathrm{Si}(\sim 7 \mathrm{ppm})$ reflecting a substantial effect of the pyridine ligand on the electronic situation of the boron atoms and the silicon atoms of the chlorine-free silyl groups. On one hand, this indicates a stronger bonding of the trimethylsilyl groups to nitrogen, on the other, this suggest that the increased coordination number of boron induces 


\begin{tabular}{llrll}
\hline & $\mathbf{2}^{\mathrm{a}}$ & $\mathbf{5}^{\mathrm{b}}$ & $\mathbf{3}^{\mathrm{a}}$ & $\mathbf{6}^{\mathrm{b}}$ \\
${ }^{\mathrm{T}} \mathrm{H}$ & $1.10\left(\mathrm{SiCl}_{2}\left(\mathrm{CH}_{3}\right)\right)$ & $0.45\left(\mathrm{SiCl}_{2}\left(\mathrm{CH}_{3}\right)\right)$ & - & - \\
${ }^{13} \mathrm{C}$ & $12.7\left(\mathrm{SiCl}_{2}\left(\mathrm{CH}_{3}\right)\right)$ & $7.12\left(\mathrm{SiCl}_{2}\left(\mathrm{CH}_{3}\right)\right)$ & - & - \\
${ }^{29} \mathrm{Si}$ & $0.25\left(\mathrm{SiCl}_{2}\left(\mathrm{CH}_{3}\right)\right)$ & $-7.83\left(\mathrm{SiCl}_{2}\left(\mathrm{CH}_{3}\right)\right)$ & $-26.5\left(\mathrm{SiCl}_{3}\right)$ & $-34.4\left(\mathrm{SiCl}_{3}\right)$ \\
${ }^{11} \mathrm{~B}$ & $7.87\left(\mathrm{NCl}_{2} B \cdot \mathrm{Py}\right)$ & $5.16\left(\mathrm{~N}_{2} \mathrm{Cl} B \cdot \mathrm{Py}\right)$ & $7.75\left(\mathrm{NCl}_{2} B \cdot \mathrm{Py}\right)$ & $4.67\left(\mathrm{~N}_{2} \mathrm{Cl} B \cdot \mathrm{Py}\right)$ \\
\hline
\end{tabular}

${ }^{a}$ Measured in $\mathrm{C}_{6} \mathrm{D}_{6}$; ${ }^{\mathrm{b}}$ measured in $\mathrm{CD}_{2} \mathrm{Cl}_{2}$. The change of solvent was necessary due to the reduced solubility of the diazadiboretidine-pyridin adducts $\mathbf{5}$ and $\mathbf{6}$. Differences in the chemical shift of the signals owing to the used solvent are not significant.

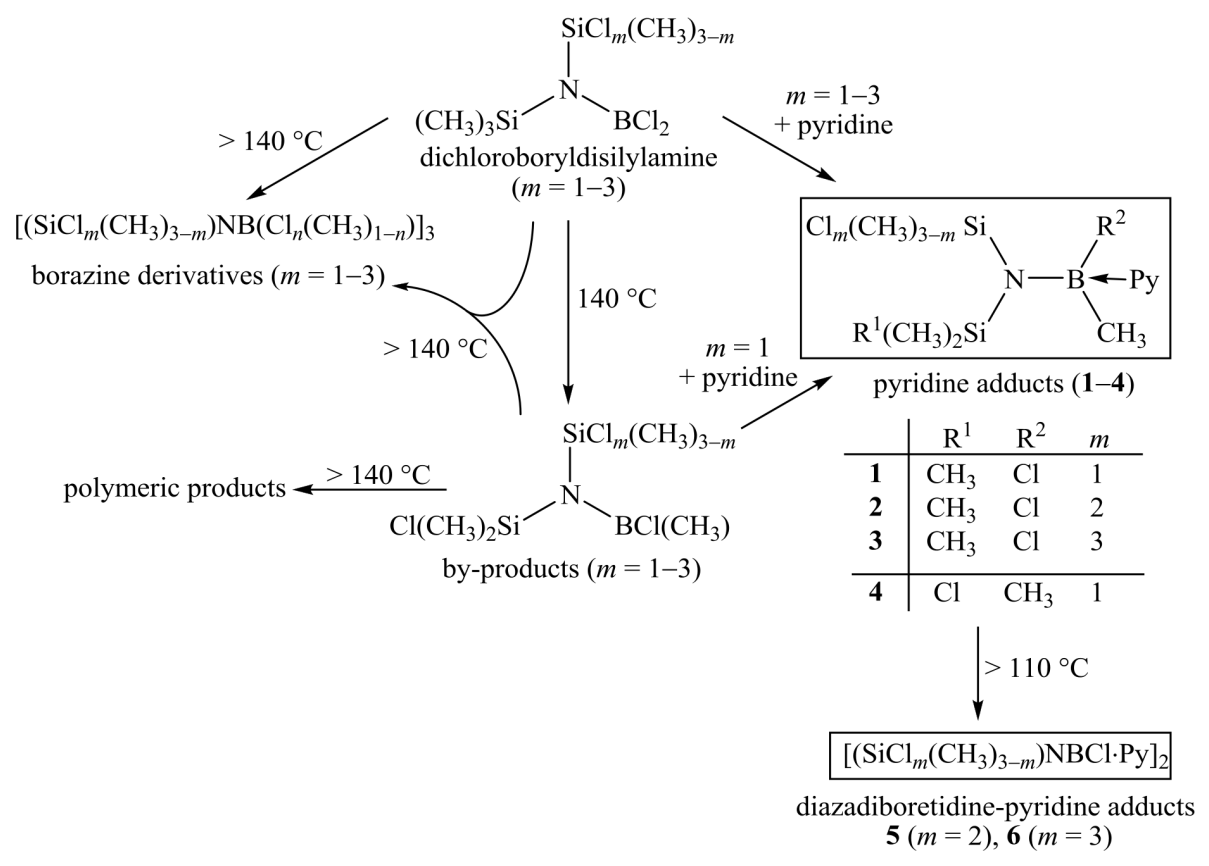

Table 1. Chemical shift (in ppm) of selected signals in the NMR spectra of compounds 2 , 3,5 , and 6 .
Scheme 1. Reaction path-
ways of dichloroboryl-
disilylamines (top, cen-
ter) during thermal treat-
ment in absence (center
and left), and presence
(right) of pyridine. a weakening of the bonds between boron and its substituents.

In contrast to the base-free chloroboryldisilylamines $[8,9,28]$, the thermal treatment of the adducts $\mathbf{1 - 4}$ at $140{ }^{\circ} \mathrm{C}$ does not lead to borazine derivatives. Instead, compounds $\mathbf{1}$ and $\mathbf{4}$ decompose without formation of detectable cyclic products, whereas the adducts $\mathbf{2}$ and $\mathbf{3}$ are converted into isolable four-membered ring diazadiboretidine-pyridine adducts $\left[\left(\mathrm{SiCl}_{m}\left(\mathrm{CH}_{3}\right)_{3-m}\right) \mathrm{NBCl} \cdot \mathrm{Py}\right]_{2}(\mathbf{5}: m=2$; 6: $m=3$ ). The cyclization reactions occur exclusively by loss of all $\mathrm{Si}\left(\mathrm{CH}_{3}\right)_{3}$ groups without any substituent exchanges, as confirmed by NMR spectroscopy. Furthermore, compared to the acyclic educts, all signals in the corresponding NMR spectra of the compounds 5 and $\mathbf{6}$ are high-field shifted (Table 1). As known from other cyclic $N$-silylated boron-nitrogen representatives obtained by silazane cleavage, such a shift is characteristic for boron nuclei, while silicon nuclei get usually deshielded during the same process $[8,9,26]$.
Single-crystal X-ray diffraction structure analyses of 5 and 6 (for details see Table 2) show planar rhombus-shaped $(\mathrm{BN})_{2}$ rings (Figs. 1 and 2). The endocyclic nitrogen atoms exhibit a slightly pyramidal coordination specified by the silyl groups which are located off the ring plane [angles between ring plane $\mathrm{B} 1-\mathrm{N} 1-\mathrm{B} 1^{\prime}-\mathrm{N} 1^{\prime}$ and a line along the $\mathrm{Si}-\mathrm{N}$ bond axis: $\left.15.9(1)^{\circ}(5), 15.8(1)^{\circ}(6)\right]$. The boron atoms are in a four-fold coordination, whereas the boronbonded chlorine atoms and the nitrogen atoms of the pyridine ligands form a plane $\mathrm{Cl1}-\mathrm{N} 2-\mathrm{Cl1}^{\prime}-\mathrm{N} 2^{\prime}$ oriented nearly perpendicular to the ring plane B1-N1$\mathrm{B} 1^{\prime}-\mathrm{N} 1^{\prime}\left(5\right.$ : $\left.89.3(1)^{\circ}, \mathbf{6}: 89.6(1)^{\circ}\right)$. The bond angles $\mathrm{B} 1-\mathrm{N} 1-\mathrm{B} 1^{\prime}$ and $\mathrm{N} 1-\mathrm{B} 1-\mathrm{N} 1^{\prime}$ within the ring units of both compounds are similar (Table 3) and, despite the presence of additional boron-bonded base, comparable to those of base-free diazadiboretidine derivatives [11-16]. However, the $\mathrm{B}-\mathrm{N}$ bond lengths of about $1.51 \AA$ in both rings are somewhat longer than those in other four-membered BN heterocycles (1.43- 
Table 2. Crystal structure data for 5 and $\mathbf{6}$.

\begin{tabular}{lll}
\hline & $\mathbf{5}$ & $\mathbf{6}$ \\
\hline Formula & $\mathrm{B}_{2} \mathrm{Si}_{2} \mathrm{Cl}_{6} \mathrm{~N}_{4} \mathrm{C}_{19.69} \mathrm{H}_{16}$ & $\mathrm{~B}_{2} \mathrm{Si}_{2} \mathrm{Cl}_{7.98} \mathrm{~N}_{4} \mathrm{C}_{19.11} \mathrm{H}_{10}$ \\
$M_{\mathrm{r}}$ & 599.14 & 656.32 \\
Crystal system & triclinic & triclinic \\
Space group & $P \overline{1}$ & $P \overline{1}$ \\
$a, \AA$ & $8.2530(6)$ & $8.2193(9)$ \\
$b, \AA$ & $8.3535(6)$ & $8.4051(9)$ \\
$c, \AA$ & $10.8041(7)$ & $10.8759(12)$ \\
$\alpha$, deg & $94.114(1)$ & $99.389(2)$ \\
$\beta$, deg & $102.530(1)$ & $97.353(2)$ \\
$\gamma$, deg & $97.617(1)$ & $98.605(2)$ \\
$V, \AA^{3}$ & 716.80 & $723.94(47)$ \\
$Z$ & 1 & 1 \\
$D_{\text {calcd }}, \mathrm{g} \mathrm{cm}{ }^{-3}$ & $1.388(1)$ & $1.505(1)$ \\
$\mu\left(\mathrm{Mo} K_{\alpha}\right), \mathrm{mm}^{-1}$ & 0.7 & 0.9 \\
$F(000), \mathrm{e}$ & 302.1 & 326.3 \\
$h k l$ range & $\pm 12, \pm 12, \pm 16$ & $\pm 12, \pm 12, \pm 16$ \\
Refl. measd. / & $11047 /$ & $11435 /$ \\
$\quad$ unique / $R_{\text {int }}$ & $5559 / 0.0229$ & $5526 / 0.0273$ \\
Param. refined & 185 & 193 \\
$R 1[I \geq 2 \sigma(I)]$ & 0.0532 & 0.0534 \\
$R 1 / w R 2($ all refl. $)$ & $0.0673 / 0.1391$ & $0.0757 / 0.1655$ \\
GoF $\left(F^{2}\right)$ & 1.035 & 1.050 \\
$\Delta \rho_{\text {fin }}($ max / & $0.90 /$ & $0.86 /$ \\
\multicolumn{1}{c}{ min $), \mathrm{e} \AA^{-3}$} & -0.70 & -0.61 \\
\hline
\end{tabular}

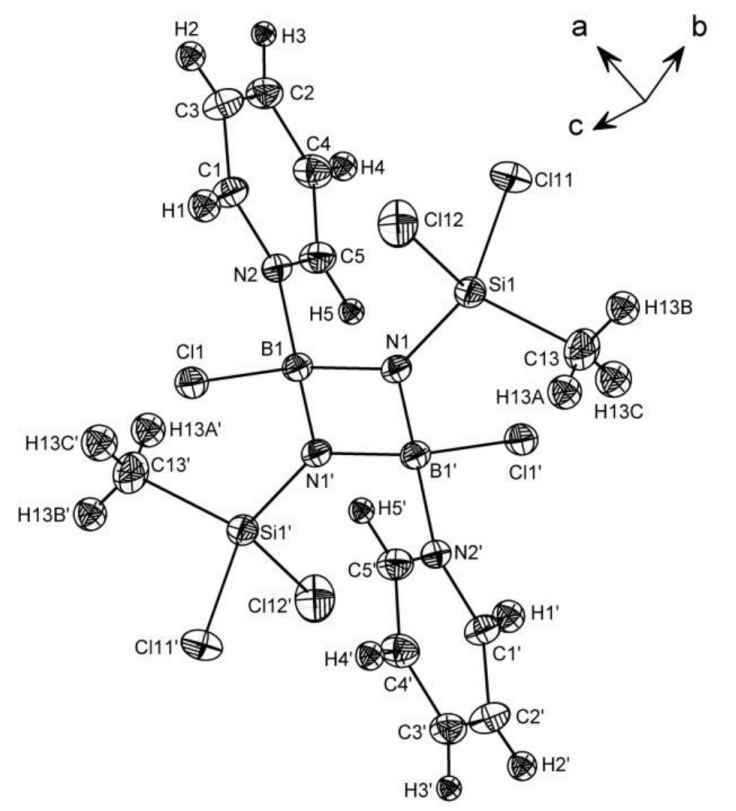

Fig. 1. Molecular structure of compound $\mathbf{5}$ (atom labeling and displacement ellipsoids at the $50 \%$ probability level for atoms refined anisotropically and $20 \%$ for hydrogen atoms are shown [symmetry code: $\left.\left.\left({ }^{\prime}\right)-x,-y, 1-z\right]\right)$.

$1.49 \AA$, for details see $[11-16])$. Additionally, the compounds presented here exhibit unusually long B$\mathrm{Cl}$ bonds $(1.9 \AA)$ compared to other known chlorine-

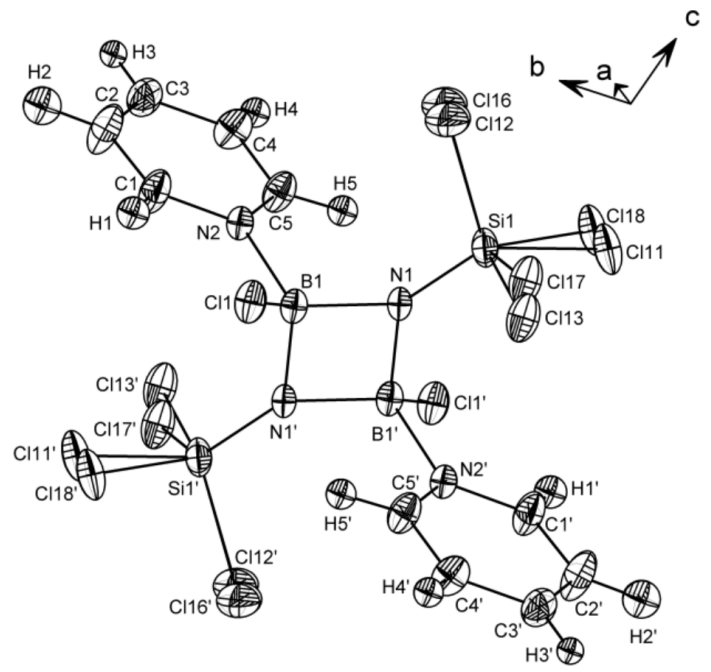

Fig. 2. Molecular structure of compound 6 (atom labeling and displacement ellipsoids at the $50 \%$ probability level for atoms refined anisotropically and $20 \%$ for hydrogen atoms are shown [symmetry code: $\left.\left.\left({ }^{\prime}\right) 1-x, 1-y, 1-z\right]\right)$.

and nitrogen-functionalized molecules like $\mathrm{BCl}_{3} \cdot \mathrm{Py}$ (1.84 $\AA$ [27]), $\mathrm{BCl}_{3} \cdot \mathrm{N}\left(\mathrm{CH}_{3}\right)_{3}(1.831 \AA$ [28] $),\left[\mathrm{Cl}_{2}\right.$ $\left.\mathrm{BN}\left(\mathrm{CH}_{3}\right)_{2}\right]_{2}(1.831 \AA[29]),\left[\left(\mathrm{Si}\left(\mathrm{CH}_{3}\right)_{3}\right) \mathrm{HNBCl}_{2}\right]_{2}$ $(1.83 \AA[30]),\left[\left(\mathrm{SiCl}\left(\mathrm{CH}_{3}\right)_{2}\right) \mathrm{HNBCl}_{2}\right]_{2}(1.81 \AA[31])$, or $\left[\mathrm{Cl}_{2} \mathrm{BNH}_{2}\right]_{3} \quad(1.845 \AA[32])$. The higher coordination number of boron in the title compounds causes an elongation of the $\mathrm{B}-\mathrm{N}$ and $\mathrm{B}-\mathrm{Cl}$ bonds. Similar effects have been observed for $\left(\mathrm{BCl}_{2}\right)_{2}$ $\mathrm{N}\left(\mathrm{NC}_{6} \mathrm{H}_{6}\right)$ [33] exhibiting boron in three- and fourfold coordination [B-Cl bond lengths: 1.759(1) $\AA$ $\left(\mathrm{NBCl}_{2}\right), 1.808(3) \AA\left(\mathrm{N}_{2} \mathrm{BCl}_{2}\right)$; $\mathrm{B}-\mathrm{N}$ bond lengths: 1.371(8) $\AA\left(\mathrm{NBCl}_{2}\right), 1.581(7) \AA\left(\mathrm{N}_{2} \mathrm{BCl}_{2}\right)$ ] [33]. Furthermore, both compounds show short $\mathrm{Si}-\mathrm{N}$ bond lengths $(\sim 1.65 \AA)$ which were neither found in basefree diazadiboretidine derivatives $(\sim 1.73 \AA[12,16])$ nor in $N$-silylated borazine derivatives $(1.73-1.80 \AA$ $[8,9,26])$.

In order to check whether pyridine-free molecules are accessible by elimination of the base through thermal decomposition, compounds 5 and $\mathbf{6}$ were subjected to TG-MS analysis. As exemplified by the TG curve of 6 (Fig. 3), both adducts start to decompose around $130{ }^{\circ} \mathrm{C}$ which is in accordance with low yields obtained after heating the reaction mixture to $140{ }^{\circ} \mathrm{C}$. Additionally, the MS signals that can be assigned to fragments of pyridine and to silyl groups in a similar temperature range indicate the destruction of the four-membered ring systems. Unfortunately, this behavior renders syntheses of pyridine-free diazadibore- 


\begin{tabular}{lcclrr}
\hline Bond length & $\mathbf{5}$ & $\mathbf{6}$ & Angle & $\mathbf{5}$ & \multicolumn{1}{c}{$\mathbf{6}$} \\
\hline B1-N1 & $1.506(2)$ & $1.508(2)$ & B1-N1-B1' & $85.62(13)$ & $86.33(13)$ \\
B1-N1 & $1.514(2)$ & $1.521(3)$ & N1-B1-N1' & $94.38(13)$ & $93.67(13)$ \\
B1-N2 & $1.617(2)$ & $1.614(2)$ & B1-N1-Si1 & $137.71(12)$ & $133.68(13)$ \\
B1-Cl1 & $1.901(2)$ & $1.891(2)$ & B1'-N1-Si1 $^{\prime}$ & $132.01(12)$ & $135.45(12)$ \\
N1-Si1 & $1.657(2)$ & $1.642(2)$ & N1-B1-C11 & $115.57(13)$ & $115.16(13)$ \\
Si1-Cl11 & $2.081(1)$ & $2.046(2)$ & N1'-B1-C11 & $114.67(12)$ & $115.18(13)$ \\
Si1-C112 & $2.061(1)$ & $1.995(1)$ & N1-B1-N2 & $112.63(14)$ & $113.57(14)$ \\
Si1-Cl13 & - & $2.126(2)$ & N1'-B1-N2 & $113.46(17)$ & $112.52(14)$ \\
Si1-C13 & $1.840(2)$ & - & N2-B1-C11 & $106.18(11)$ & $106.65(12)$ \\
\hline
\end{tabular}

Table 3. Selected bond lengths $(\AA)$, and angles (deg) for $\mathbf{5}$ and $\mathbf{6}$ with estimated standard deviations in parentheses ${ }^{\mathrm{a}}$.

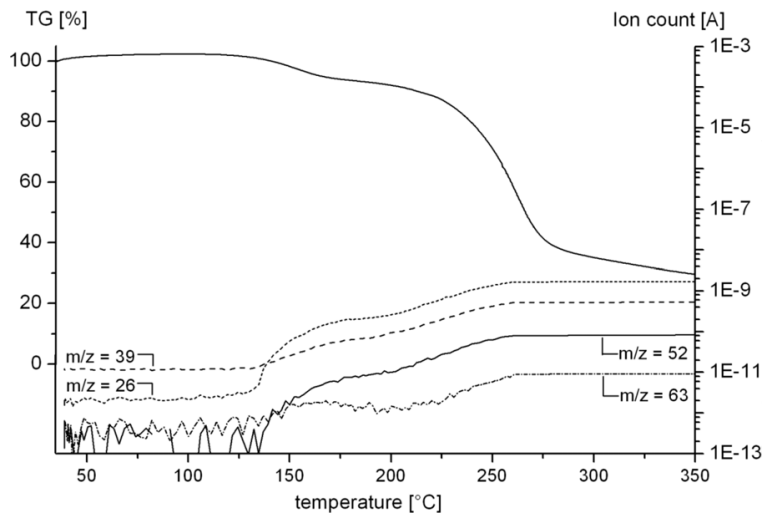

Fig. 3. Decomposition of $\mathbf{6}$ monitored by TG analysis and MS (pyridine: $m / z=52\left[\mathrm{C}_{4} \mathrm{H}_{4}\right]^{+}, m / z=39\left[\mathrm{C}_{3} \mathrm{H}_{3}\right]^{+}, m / z=$ $\left.26\left[\mathrm{C}_{2} \mathrm{H}_{2}\right]^{+} ; \mathrm{SiCl}_{3}: m / z=63[\mathrm{SiCl}]^{+}\right)$.

tidine derivatives through thermal treatment of their complexes impossible.

\section{Conclusion}

In comparison with the base-free chloroboryldisilylamines, their pyridine adducts show an enhanced reactivity, since the weak $\mathrm{B}-\mathrm{Cl}$ bonds favor the silazane cleavage significantly. The coordinative bond between the strong base and the boron atoms appears to be retained during the cyclization step inhibiting any substituent exchange by blocking the free coordination site of the boron centers. Furthermore, pyridine acts as a space-demanding ligand leading to the formation of four-membered diazadiboretidine-pyridine adducts instead of six-membered borazine derivatives.

\section{Experimental Section}

\section{Characterization techniques}

Solution NMR spectroscopic measurements were carried out at an Avance DPX-300 SB spectrometer (Bruker) equipped with a $300 \mathrm{MHz}$ magnet $\left(B_{0}=7.05 \mathrm{~T}\right)$ working at $300.13 \mathrm{MHz}\left({ }^{1} \mathrm{H}\right.$ NMR), $75.46 \mathrm{MHz}\left({ }^{13} \mathrm{C}\right.$ NMR $), 96.29 \mathrm{MHz}$ $\left({ }^{11} \mathrm{~B}\right.$ NMR), or $59.63 \mathrm{MHz}\left({ }^{29} \mathrm{Si}\right.$ NMR). IR spectra were recorded on a Bruker IFS $113 \mathrm{v}$ FT-IR spectrometer in the range between 400 and $4000 \mathrm{~cm}^{-1}$. Mass spectra were obtained using a mass spectrometer GCMS-QP2010 Plus (Shimadzu) with electron impact ionization $(70 \mathrm{eV})$. The decomposition of the diazadiboretidine-pyridine adducts was examined using thermogravimetric analysis (TG) in a Netzsch STA409 instrument (heating rate $10 \mathrm{~K} \mathrm{~min}^{-1}$, argon flow) equipped with a Balzer QMS421 quadrupole mass spectrometer.

\section{Syntheses}

All reactions were performed under an inert argon atmosphere in carefully dried glassware and solvents. Pyridine (Merck) was dried over molecular sieves (Merck), toluene (Merck) and $m$-xylene (Acros Organics) were distilled over sodium/benzophenone (Merck). Reactants $\left[\mathrm{SiCl}\left(\mathrm{CH}_{3}\right)_{2}\right] \mathrm{N}\left[\mathrm{Si}\left(\mathrm{CH}_{3}\right)_{3}\right]\left(\mathrm{BCl}_{2}\right)$ [8], $\left[\mathrm{SiCl}_{2}\left(\mathrm{CH}_{3}\right)\right]-$ $\mathrm{N}\left[\mathrm{Si}\left(\mathrm{CH}_{3}\right)_{3}\right]\left(\mathrm{BCl}_{2}\right)$ [9], $\left(\mathrm{SiCl}_{3}\right) \mathrm{N}\left[\mathrm{Si}\left(\mathrm{CH}_{3}\right)_{3}\right] \mathrm{N}\left(\mathrm{BCl}_{2}\right)$ [8] and [ $\left.\mathrm{SiCl}\left(\mathrm{CH}_{3}\right)_{2}\right]_{2} \mathrm{~N}\left[\mathrm{BCl}\left(\mathrm{CH}_{3}\right)\right]$ [8] were prepared according to the literature.

\section{Compounds 1-4}

In a typical experiment, $0.006 \mathrm{~mol}$ of the corresponding dichloroboryldisilylamine or of chloromethylboryl-bischlorodimethylsilyl-amine was dissolved in $10 \mathrm{~mL}$ toluene. The drop- wise addition of $0.5 \mathrm{~mL}(0.0062 \mathrm{~mol})$ pyridine to this solution at $r$.t. led to colorless precipitates which dissolved on heating to $110^{\circ} \mathrm{C}$. After $4 \mathrm{~h}$ in boiling toluene the reaction mixture was cooled to r. t., followed by the removal of excess pyridine and toluene in vacuum. The obtained raw products 1-4 were recrystallized from toluene and subsequently dried at $45{ }^{\circ} \mathrm{C}$ in dynamic vacuum over night.

Compound 1: Yield: $1.68 \mathrm{~g}(82 \%) .-{ }^{1} \mathrm{H}$ NMR $\left(\mathrm{C}_{6} \mathrm{D}_{6}\right)$ : $\delta=0.48\left(\mathrm{Si}\left(\mathrm{CH}_{3}\right)_{3}, 9 \mathrm{H}\right), 0.71\left(\mathrm{SiCl}\left(\mathrm{CH}_{3}\right)_{2}, 6 \mathrm{H}\right), 6.29$ $(\mathrm{m}, 2 \mathrm{H}$ in meta position, pyridine), $6.60(\mathrm{~m}, 1 \mathrm{H}$ in para position, pyridine), 8.98 (m, $2 \mathrm{H}$ in ortho position, pyridine $).{ }^{13} \mathrm{C}$ NMR $\left(\mathrm{C}_{6} \mathrm{D}_{6}\right): \delta=5.62\left(\left(\mathrm{Si}\left(\mathrm{CH}_{3}\right)_{3}\right)\right.$, $8.96\left(\mathrm{SiCl}\left(\mathrm{CH}_{3}\right)_{2}\right), 125.5$ (meta position, pyridine), 141.9 (para position, pyridine), 144.8 (ortho position, pyridine). ${ }^{11} \mathrm{~B}$ NMR $\left(\mathrm{C}_{6} \mathrm{D}_{6}\right): \delta=8.42 .-{ }^{29} \mathrm{Si} \mathrm{NMR}: \delta=13.9$ $\left(\mathrm{Si}\left(\mathrm{CH}_{3}\right)_{3}\right), 5.61\left(\mathrm{SiCl}\left(\mathrm{CH}_{3}\right)_{2}\right) .-\mathrm{IR}(\mathrm{film}): \tilde{v}=3114(\mathrm{vw})$, 3064 (vw), 298 (w), 2960 (w), 2901 (vw), 1626 (m), 
1575 (w), 1489 (m), 1459 (st), 1413 (w), 1267 (st), 1258 (st), 1212 (w), 1159 (w), 1104 (st), 1087 (vst), 1066 (st), 1045 (vst), 1022 (m), 931 (vst), 873 (st), 846 (st), 822 (st), $794(\mathrm{~m}), 775$ (m), 753 (w), 728 (m), 711 (w), 687 (vst), $644(\mathrm{w}), 629(\mathrm{vw}), 615(\mathrm{w}), 593(\mathrm{w}), 584(\mathrm{w}), 475(\mathrm{st})$, 467 (st), $431(\mathrm{w}) \mathrm{cm}^{-1}$. - MS (EI, $\left.70 \mathrm{eV}\right): \mathrm{m} / z(\%)=342(0)$, 248 (15), 226 (2), 210 (1), 138 (3), 118 (100), 98 (11), 93 (19), 88 (5), 79 (38), 73 (27), 63 (5), 52 (29), 43 (4), 39 (4), 26 (4).

Compound 2: Yield: $1.95 \mathrm{~g}(90 \%) .-{ }^{1} \mathrm{H}$ NMR $\left(\mathrm{C}_{6} \mathrm{D}_{6}\right)$ : $\delta=0.43\left(\mathrm{Si}\left(\mathrm{CH}_{3}\right)_{3}, 9 \mathrm{H}\right), 1.10\left(\mathrm{SiCl}_{2}\left(\mathrm{CH}_{3}\right), 3 \mathrm{H}\right), 6.25(\mathrm{~m}$, $2 \mathrm{H}$ in meta position, pyridine), 6.57 ( $\mathrm{m}, 1 \mathrm{H}$ in para position, pyridine), 8.955 (m, $2 \mathrm{H}$ in ortho position, pyridine). ${ }^{13} \mathrm{C}$ NMR $\left(\mathrm{C}_{6} \mathrm{D}_{6}\right): \delta=5.28\left(\left(\mathrm{Si}\left(\mathrm{CH}_{3}\right)_{3}\right), 12.7\left(\mathrm{SiCl}_{2}\left(\mathrm{CH}_{3}\right)\right)\right.$, 125.7 (meta position, pyridine), 142.3 (para position, pyridine), 144.5 (ortho position, pyridine). $-{ }^{11} \mathrm{~B}$ NMR $\left(\mathrm{C}_{6} \mathrm{D}_{6}\right)$ : $\delta=7.87 .{ }^{29} \mathrm{Si}$ NMR $\left(\mathrm{C}_{6} \mathrm{D}_{6}\right): \delta=7.95\left(\mathrm{Si}\left(\mathrm{CH}_{3}\right)_{3}\right), 0.25$ $\left(\mathrm{SiCl}_{2}\left(\mathrm{CH}_{3}\right)\right)$. - IR (film): $\tilde{v}=3115$ (vw), 3065 (vw), 2987 (vw), 2956 (vw), 2900 (vw), 1629 (w), 1575 (vw), 1489 (w), 1460 (st), 1413 (w), 1263 (m), 1250 (m), 1214 (w), 1160 (w), 1104 (m), 1092 (st), 1066 (m), 1038 (vst), 1023 (m), 943 (vst), 932 (vst), 860 (vst), 848 (vst), 799 (m), $777(\mathrm{~m}), 751(\mathrm{~m}), 742(\mathrm{~m}), 708$ (w), 688 (st), 646 (vw), 630 (vw), 619 (vw), 601 (vw), 587 (vw), $523(\mathrm{~m}), 486(\mathrm{~m})$, $468(\mathrm{w}), 435(\mathrm{vw}) \mathrm{cm}^{-1}$. - MS (EI, $\left.70 \mathrm{eV}\right): \mathrm{m} / z(\%)=$ 362 (0), 268 (100), 248 (5), 158 (3), 138 (62), 118 (71), 113 (11), 93 (67), 88 (12), 79 (64), 73 (9), 63 (15), 52 (36), 43 (8), 39 (7), 26 (11).

Compound 3: Yield: $2.01 \mathrm{~g}(88 \%) .-{ }^{1} \mathrm{H}$ NMR $\left(\mathrm{C}_{6} \mathrm{D}_{6}\right)$ : $\delta=0.54\left(\mathrm{Si}\left(\mathrm{CH}_{3}\right)_{3}, 9 \mathrm{H}\right), 6.29(\mathrm{~m}, 2 \mathrm{H}$ in meta position, pyridine), $6.62(\mathrm{~m}, 1 \mathrm{H}$ in para position, pyridine), 8.87 ( $\mathrm{m}, 2 \mathrm{H}$ in ortho position, pyridine). $-{ }^{13} \mathrm{C}$ NMR $\left(\mathrm{C}_{6} \mathrm{D}_{6}\right)$ : $\delta=9.17\left(\left(\mathrm{Si}\left(\mathrm{CH}_{3}\right)_{3}\right), 125.9\right.$ (meta position, pyridine), 142.6 (para position, pyridine), 144.4 (ortho position, pyridine). ${ }^{11} \mathrm{~B}$ NMR $\left(\mathrm{C}_{6} \mathrm{D}_{6}\right): \delta=7.75 .{ }^{29} \mathrm{Si} \mathrm{NMR}\left(\mathrm{C}_{6} \mathrm{D}_{6}\right): \delta=9.17$ $\left(\mathrm{Si}\left(\mathrm{CH}_{3}\right)_{3}\right),-26.5\left(\mathrm{SiCl}\left(\mathrm{CH}_{3}\right)_{2}\right)$. - IR (film): $\tilde{v}=3116(\mathrm{vw})$, 3065 (vw), 2991 (vw), 2959 (vw), 2899 (vw), 1626 (m), 1576 (vw), 1492 (w), 1463 (st), 1410 (w), 1272 (m), 1253 (m), 1214 (w), 1165 (w), 1105 (m), 1091 (st), 1064 (m), 1031 (vst), 1023 (vst), 943 (vst), 852 (vst), 776 (m), 743 (m), $732(\mathrm{~m}), 703$ (w), $686(\mathrm{~m}), 632$ (m), 592 (m), 563 (vst), 492 (st), 470 (w), 430 (w) $\mathrm{cm}^{-1}$. - MS (EI, $\left.70 \mathrm{eV}\right): \mathrm{m} / \mathrm{z}$ $(\%)=382$ (0), 288 (27), 268 (2), 158 (7), 138 (100), 133 (4), 118 (18), 113 (15), 93 (38), 88 (10), 79 (73), 73 (7), 63 (18), 52 (55), 43 (12), 39 (7), 26 (8).

Compound 4: Yield: $1.60 \mathrm{~g}(78 \%) .-{ }^{1} \mathrm{H}$ NMR $\left(\mathrm{C}_{6} \mathrm{D}_{6}\right)$ : $\delta=0.66\left(\mathrm{SiCl}\left(\mathrm{CH}_{3}\right)_{2}, 12 \mathrm{H}\right), 0.83\left(\mathrm{BCl}\left(\mathrm{CH}_{3}\right), 3 \mathrm{H}\right), 6.37$ ( $\mathrm{m}, 2 \mathrm{H}$ in meta position, pyridine), $6.64 \mathrm{~m}, 1 \mathrm{H}$ in para position, pyridine), $8.97(\mathrm{~m}, 2 \mathrm{H}$ in ortho position, pyridine). $-{ }^{13} \mathrm{C} \mathrm{NMR}\left(\mathrm{C}_{6} \mathrm{D}_{6}\right): \delta=8.94\left(\mathrm{Si}\left(\mathrm{CH}_{3}\right)_{3}\right)$, signal of $\left(\mathrm{BCl}\left(\mathrm{CH}_{3}\right)\right)$ too broad, 125.6 (meta position, pyridine), 140.9 (para position, pyridine), 144.9 (ortho position, pyridine). ${ }^{11}$ B NMR: $\left(\mathrm{C}_{6} \mathrm{D}_{6}\right) \delta=9.76 .{ }^{29} \mathrm{Si} \mathrm{NMR}\left(\mathrm{C}_{6} \mathrm{D}_{6}\right): \delta=15.4$. -
IR (film): $\tilde{v}=3112$ (vw), 3062 (vw), 2995 (vw), $2974(\mathrm{w})$, 2942 (vw), 2905 (vw), 1623 (st), 1575 (vw), 1487 (m), 1457 (vst), 1417 (m), 1312 (st), 1270 (vst), 1262 (vst), 1215 (w), 1160 (w), 1091 (st), 1079 (vst), 1053 (vst), 998 (vst), 910 (vst), 887 (vst), 826 (st), 811 (st), 791 (m), 770 (st), 706 (m), 693 (st), 680 (m), 629 (w), 617 (st), $563(\mathrm{~m}), 508$ (w), 478 (st), 465 (st), $440(\mathrm{~m}), 419$ (vw), $408(\mathrm{~m}) \mathrm{cm}^{-1}$. - MS (EI, $\left.70 \mathrm{eV}\right): \mathrm{m} / z(\%)=342(0)$, 248 (24), 226 (7), 210 (3), 138 (2), 118 (100), 98 (17), 93 (17), 88 (5), 79 (45), 73 (27), 68 (19), 65 (10), 63 (7), 52 (36), 43 (3), 39 (5), 26 (5).

\section{Decomposition of the compounds $\mathbf{1}-\mathbf{4}$}

$0.004 \mathrm{~mol}$ of the corresponding pyridine adduct $(\mathbf{1}-\mathbf{4})$ was dissolved in $20 \mathrm{~mL} m$-xylene and heated to $140{ }^{\circ} \mathrm{C}$ for $1-$ $2 \mathrm{~d}$. Dark-brown solutions were obtained from which compounds 5 (from $\mathbf{3}$ ) and $\mathbf{6}$ (from 4 ) crystallized in the form of colorless rhombus-shaped crystals by cooling to $r$. t. The raw products were isolated by removing the solvent and volatile side products in vacuum resulting in brownish solids which were recrystallized from toluene and dried in dynamic vacuum at $45{ }^{\circ} \mathrm{C}$.

Compound 5: Yield: $0.55 \mathrm{~g}(54 \%) .-{ }^{1} \mathrm{H} \mathrm{NMR}\left(\mathrm{CD}_{2} \mathrm{Cl}_{2}\right)$ : $\delta=0.45\left(\mathrm{SiCl}_{2}\left(\mathrm{CH}_{3}\right), 6 \mathrm{H}\right), 7.78(\mathrm{~m}, 4 \mathrm{H}$ in meta position, pyridine), 8.21 ( $\mathrm{m}, 2 \mathrm{H}$ in para position, pyridine), 9.35 ( $\mathrm{m}$, $4 \mathrm{H}$ in ortho position, pyridine). $-{ }^{13} \mathrm{C}$ NMR $\left(\mathrm{CD}_{2} \mathrm{Cl}_{2}\right): \delta=$ $7.12\left(\mathrm{SiCl}_{2}\left(\mathrm{CH}_{3}\right)\right), 126.2$ (meta position, pyridine), 143.2 (para position, pyridine), 146.1 (ortho position, pyridine). ${ }^{11} \mathrm{~B}$ NMR $\left(\mathrm{CD}_{2} \mathrm{Cl}_{2}\right): \delta=5.16 .{ }^{29} \mathrm{Si}$ NMR $\left(\mathrm{CD}_{2} \mathrm{Cl}_{2}\right): \delta=$ -7.83. - IR (film): $\tilde{v}=3130(\mathrm{w}), 3117(\mathrm{w}), 3065(\mathrm{w})$, 1625 (m), 1575 (s), 1486 (m), 1456 (vst), 1408 (vw), 1401 (vw), 1343 (vw), 1262 (m), 1213 (st), 1184 (vst), 1173 (vst), 1101 (m), 1084 (st), 1061 (s), 1026 (m), 988 (m), 977 (m), 968 (vst), 958 (vst), 889 (w), 874 (m), 863 (st), 793 (vst), 779 (st), 758 (vst), 722 (m), 689 (st), 657 (m), $67(\mathrm{~m}), 511(\mathrm{st}), 490(\mathrm{~m}), 463(\mathrm{~m}), 47(\mathrm{st}) \mathrm{cm}^{-1}$. - MS (EI, $70 \mathrm{eV}): m / z(\%)=507$ (0), 392 (1), 348 (4), 333 (30), 313 (3), 183 (6), 163 (13), 138 (19), 113 (11), 88 (7), 79 (100), 63 (7), 52 (76), 39 (10), 39 (25), 26 (10).

Compound 6: Yield: $0.47 \mathrm{~g}(43 \%) .-{ }^{1} \mathrm{H} \mathrm{NMR}\left(\mathrm{CD}_{2} \mathrm{Cl}_{2}\right)$ : $\delta=7.83(\mathrm{~m}, 4 \mathrm{H}$ in meta position, pyridine), 8.25 (m, $2 \mathrm{H}$ in para position, pyridine), 9.48 ( $\mathrm{m}, 4 \mathrm{H}$ in ortho position, pyridine). $-{ }^{13} \mathrm{C} \mathrm{NMR}\left(\mathrm{CD}_{2} \mathrm{Cl}_{2}\right): \delta=126.5$ (meta position, pyridine), 143.8 (para position, pyridine), 146.0 (ortho position, pyridine). $-{ }^{11} \mathrm{~B} \mathrm{NMR}\left(\mathrm{CD}_{2} \mathrm{Cl}_{2}\right): \delta=$ 4.67. $-{ }^{29} \mathrm{Si} \mathrm{NMR}\left(\mathrm{CD}_{2} \mathrm{Cl}_{2}\right): \delta=-34.4$. $-\mathrm{IR}$ (film): $\tilde{v}=$ 3132 (w), 3119 (w), 3067 (w), $1626(\mathrm{~m}), 1575(\mathrm{w}), 1488(\mathrm{~m})$, 1458 (vst), 1411 (vw), 1398 (vw), 1344 (vw), 1214 (m), 1185 (vst), 1162 (vst), 1101 (m), 1085 (st), 1061 (w), 1026 (m), 986 (m), 975 (st), 963 (st), 949 (vst), 902 (w), 869 (w), 850 (m), 804 (st), 798 (st), 783 (st), 763 (st), 678 (st), 660 (m), 647 (m), 589 (vst), 557 (vst), 493 (m), 472 (st), 441 (st) $\mathrm{cm}^{-1}$. - MS (EI, $\left.70 \mathrm{eV}\right): \mathrm{m} / z(\%)=548(0)$, 
432 (7), 390 (7), 353 (35), 183 (17), 160 (22), 158 (16), 133 (6), 123 (4), 113 (7), 88 (8), 79 (100), 63 (7), 52 (75), 39 (10), 26 (19).

\section{Crystal structure analyses}

Data collection $(T=100 \mathrm{~K})$ for single-crystal diffraction structure analysis was performed on a Smart APEX I three-circle single-crystal diffractometer (Bruker) equipped with a CCD-detector using $\operatorname{Mo} K_{\alpha}$ radiation $(\lambda=0.71073 \AA$ ). The data reduction was carried out with the BRUKER SUITE software package [34], and the semi-empirical correction of the intensities for absorption effects by using the program SADABS [35]. The structure was solved by Direct Methods. For the structure solution as well as for the refinement the SHELXTL software package [36] was used. Table 2 gives data of crystallographic measurements of compounds 5 and $\mathbf{6}$. All hydrogen atoms were found and refined isotropically, whereas $\mathrm{Cl11}, \mathrm{Cl1} 2$ and $\mathrm{Cl13}$ showed some positional disorder creating split positions Cl16, Cl17 and Cl18. Some carbon atoms of solvate molecules were refined isotropically.

CCDC 842318 (5), and CCDC 842319 (6) contain the supplementary crystallographic data for this paper. These data can be obtained free of charge from The Cambridge Crystallographic Data Centre via www.ccdc.cam.ac.uk/data_ request/cif.

\section{Acknowledgements}

We thank Dr. F. Gruber and Dr. H. Nuss for singlecrystal measurements, J. Wilfert for TG analyses, and M.-L. Schreiber for supporting analytical work.
[1] H.-P. Baldus, O. Wagner, M. Jansen, Mat. Res. Soc. Symp. Proc. 1992, 271, 821.

[2] M. Jansen, B. Jäschke, T. Jäschke, Struct. Bond. 2002, 102, 137.

[3] J. Haberecht, F. Krumeich, H. Grützmacher, R. Nesper, Chem. Mater. 2004, 16, 418.

[4] J. Haberecht, R. Nesper, H. Grützmacher, Chem. Mater. 2005, 17, 2340.

[5] T. Jäschke, M. Jansen, J. Eur. Ceram. Soc. 2005, 25, 211.

[6] T. Jäschke, M. Jansen, J. Mater. Chem. 2006, 16, 2792.

[7] A. Epple, Dissertation, University of Stuttgart, Stuttgart (Germany) 2006.

[8] F. Schurz, M. Jansen, Z. Anorg. Allg. Chem. 2010, 636, 1199.

[9] F. Schurz, unpublished results.

[10] K.-H. van Bonn, T. v. Bennigsen-Mackiewicz, J. Kiesgen, C. v. Plotho, P. Paetzold, Z. Naturforsch. 1988, $43 b, 61$.

[11] P. Paetzold, A. Richter, Th. Thijssen, S. Würtenberg, Chem. Ber. 1979, 112, 3811.

[12] H. Hess, Acta Crystallogr. 1969, B25, 2342.

[13] H. Nöth, M. Schwartz, S. Weber, Chem. Ber. 1985, 118, 4716.

[14] P. Paetzold, E. Schröder, G. Schmid, R. Boese, Chem. Ber. 1985, 118, 3205.

[15] E. v. Steuber, G. Elter, M. Noltemeyer, H.-G. Schmidt, A. Meller, Organometallics 2000, 19, 5083.

[16] C. Matthes, U. Klingebiel, S. Deuerlein, H. Ott, D. Stalke, Z. Anorg. Allg. Chem. 2008, 634, 2402.

[17] H. S. Turner, R. J. Warne, Adv. Chem. Ser. 1964, 42, 290; H. Nöth, Z. Naturforsch. 2009, 64b, 1090.

[18] P. T. Clarke, H. M. Powell, J. Chem. Soc. B 1966, 1172.

[19] B. Thiele, P. Schreyer, U. Englert, P. Paetzold, R. Boese, B. Wrackmeyer, Chem. Ber. 1991, 124, 2209.

[20] C. R. Russ, A. G. MacDiarmid, Angew. Chem. 1964, 76, 500; Angew. Chem., Int. Ed. Engl. 1964, 3, 509.
[21] P. Geymayer, E. G. Rochow, U. Wannagat, Angew. Chem. 1964, 76, 499; P. Geymayer, E. G. Rochow, U. Wannagat, Angew. Chem., Int. Ed. Engl. 1964, 3, 633.

[22] P. Geymayer, E. G. Rochow, Monatsh. Chem. 1964, 97, 429.

[23] H.-A. Steuer, A. Meller, G. Elter, J. Organomet. Chem. 1985, 295, 1.

[24] P. Paetzold, J. Kiesgen, K. Krahé, H.-U. Meier, R. Boese, Z. Naturforsch. 1991, 46b, 853.

[25] B.-L. Li, M. A. Goodman, R. H. Neilson, Inorg. Chem. 1984, 23, 1368

[26] B. Anand, H. Nöth, H. Schwenk-Kircher, A. Troll, Eur. J. Inorg. Chem. 2008, 3186.

[27] K. Töpel, K. Hensen, M. Trömel, Acta Crystallogr. 1990, $B 37,969$.

[28] H. Hess, Acta Crystallogr. 1969, B25, 2338.

[29] H. Hess, Z. Kristallogr. 1963, 118, 361.

[30] M. Jansen, T. Jäschke, Z. Anorg. Allg. Chem. 1999, 625, 1957.

[31] U. Müller, M. Weinmann, M. Jansen, J. Mater. Chem. 2008, 18,3671 .

[32] H. Hess, D. Lux, W. Schwarz, Z. Naturforsch. 1977, $32 b, 982$.

[33] L. M. Engelhardt, G. E. Jacobsen, P. C. Junk, C. L. Raston, A. H. White, J. Chem. Soc., Chem. Commun. 1990, 89.

[34] BRUKER SUITE (version 2008/3), Bruker Analytical X-ray Instruments Inc., Madison, Wisconsin (USA) 2008.

[35] G. M. Sheldrick, SADABS (version 2008/1), Program for Empirical Absorption Correction of Area Detector Data, University of Göttingen, Göttingen (Germany) 2008.

[36] G. M. Sheldrick, Acta Crystallogr. 2008, A64, 112. 